\title{
IDAS Y VENIDAS DE LA DESCENTRALIZACIÓN DEL ESTADO EN AMÉRICA LATINA: EL CASO URUGUAYO
}

\author{
Alejandro Noboa Silva, ${ }^{1}$ Iván Sánchez ${ }^{2}$ y Mariano Suárez Elías ${ }^{3}$
}

Resumen. Al principio se recorren las diferentes teorías que alumbran las experiencias de descentralización en América Latina, fenómeno que aparece tardíamente en Uruguay. Más adelante se muestran elementos para la evaluación de políticas de descentralización del gobierno central del Uruguay. Allí se presentan los esfuerzos del Ministerio de Ganadería, del Ministerio del Interior y del Ministerio de Desarrollo Social. Se relevan percepciones de los actores participantes y se concluye en consecuencia sobre la marcha de estas políticas sus aspectos positivos y negativos tales como el funcionamiento de la nueva institucionalidad, relaciones con otras instituciones, actores participantes, logros obtenidos y obstáculos detectados.

Palabras clave: descentralización, políticas públicas, gobernanza, relaciones interinstitucionales, participación.

Abstract. At first, the different theories that enlighten the experience of decentralization in Latin America are explored, a phenomenon that appears belatedly in Uruguay. Below are elements for the evaluation of decentralization policies of the central government of Uruguay. There the efforts of the Ministry of Livestock and Agriculture, the Ministry of the Interior and the Ministry of Social Development are presented. Perceptions of the participating actors are exported and the positive and negative aspects such as the functioning of the new institutionality, relations with other institutions, participating actors, achieved achievements and obstacles detected are consequently concluded on the progress of these policies.

Keywords: decentralization, public policy, governance, interinstitutional relations, participation.

\footnotetext{
${ }^{1}$ Sociólogo, Doctor en Ciencia Política y Sociología por la Universidad Complutense de Madrid. Docente de alta dedicación en la Universidad de la República Sede Salto (Uruguay). Coordinador del GEPADE (Grupo de Estudios de la Participación y la Descentralización). Rivera 1350, Salto, Uruguay. Correo electrónico: anoboa@unorte.edu.uy

${ }^{2}$ Licenciado en Ciencia Política, Magister en Estudios Contemporáneos de America Latina (UdelaR y Universidad Complutense de Madrid), trabajo en Programa Uruguay Integra (DDIP-OPP Presidencia de la República). Integrante del Grupo de Estudios de la Participación y la Descentralización (GEPADE). Rivera 1350, - Salto Uruguay. Correo electrónico: sanchez.82@gmail.com

${ }^{3}$ Licenciado en Ciencias Sociales, Magíster en Desarrollo Humano (FLACSO-Argentina) y Candidato a Doctor en Estudios Sociales de América Latina (UNC-Argentina). Docente de dedicación total en la Universidad de la República, Sede Salto (Uruguay). Integrante del Grupo de Estudios de la Participación y la Descentralización (GEPADE). Rivera 1350 Salto, Uruguay. . Correo electrónico: marianodoc01@unorte.edu.uy
} 


\section{Introducción}

El fracaso del modelo neoliberal en América Latina, condujo a un descontento que favoreció al ascenso democrático de gobiernos de izquierda. Dichos gobiernos apuestan a un modelo neo-desarrollista y a profundizar las democracias, por lo que aparece la necesidad de un Estado presente en lo económico y social, y en el que la descentralización y los mecanismos de participación son claves para elaborar políticas en función de las necesidades populares, y para el control ciudadano, y la intersectorialidad para dar respuestas integrales, y en clave de derecho, a problemas complejos de poblaciones cada vez más exigentes (Cunill Grau, 2014).

Los casos que se presentan en este trabajo, las Mesas Interinstitucionales de Políticas Sociales, las Mesas de Desarrollo Rural y las Mesas de Seguridad Ciudadana en los departamentos de Artigas, Paysandú y Salto, son reflejo de ello, y no sólo pertenecen a diferentes regiones socio-productivas, sino que además, su implementación se desarrolló en el marco de gestiones de gobierno que si bien responden centralmente a una misma fuerza política, departamentalmente están contextualizadas por diferentes gobiernos partidarios, en los que están representados los tres partidos mayoritarios del país, en este artículo veremos similitudes y diferencias entre ellas.

El análisis de las tres entidades está basado en una investigación desarrollada durante el 2015, realizada a partir de la aplicación de un diseño cualitativo donde se realizó una entrada exploratoria a campo, en la cual se realizaron dos entrevistas por cada una de las tres entidades y para cada departamento (total: 18).

El objetivo de esta entrada exploratoria fue identificar aspectos relevantes y característicos de cada una de las mesas para poder tener una primera impresión y visualización de su funcionamiento. El análisis de estos hallazgos exploratorios permitió la elaboración de las pautas para las entrevistas semi-estructuradas con las que se continuó la indagación.

Las entrevistas semi-estructuradas fueron un total de 48 para los tres departamentos, Artigas, Salto y Paysandú. ). En todos los casos las entrevistas se realizaron a los representantes de las instituciones y organizaciones que estaban encargados de asistir a las reuniones.

\section{Contexto histórico y teórico de la Transformación Democrática del Estado en Uruguay.}




\subsection{Modelo de desarrollo y tipo de Estado y sociedad en América Latina}

La relación entre modelo de desarrollo y tipo de Estado y sociedad, ha sido clara en Latinoamérica, lo que nos permite repasar el comportamiento de las diadas centralización-descentralización, sectorialidad-intersectorialidad, y representaciónparticipación.

1. El modelo desarrollista (1930-1970) nace con la caída de la demanda de commodities latinoamericanos, llevando a balanzas comerciales desfavorables y escasez de divisas. Los gobiernos latinoamericanos comenzaron a controlar importaciones de bienes y exportaciones de capital, irrumpiendo la necesidad de industrializar para el mercado interno. El modelo perseguía la modernización mediante la industrialización, la cual requirió del rol activo del Estado como dirigista y empresario, que permitía acceso a créditos e insumos de funcionamiento baratos para el sector industrial.

El Estado, en esta etapa, se desarrolló con una lógica vertical y sectorial, y un corporativismo en su relación con la sociedad. Lo subnacional se concebía como resabio de sociedades premodernas, por tanto amenaza para la modernización y su pretensión uniformizante (Moreira y Veneziano, 1995).

La centralización sometía lo subnacional al control sobre competencias y recursos, asignándole funciones administrativas o subsidiarias (Veneziano, 2009). Ese centro se estructuraba en sectores especializados, en el que cada uno según su racionalidad y sin comunicación entre sí, se extendía por todo el territorio nacional uniformemente.

2. El modelo neoliberal (1970-2000) tuvo su fundamento ideológico en el Consenso de Washington, y lo central era apostar al mercado como motor de crecimiento. La idea era que al reducir el Estado, aumentarían las inversiones, y así el empleo y consumo. Reducir el Estado además, era reducir el principal obstáculo para oportunidades particulares.

El éxito no sería responsabilidad de la política, sino responsabilidad individual (Garretón, 2004). El modelo se sostuvo en el fortalecimiento de sistemas presidencialistas, en el desembarco de tecnócratas con libertad de acción en altas esferas de los poderes ejecutivos, y el no reconocimiento de formas de representación fuera de lo institucional (Veneziano, 2009).

Tanto la descentralización como la participación no se relacionan en esta visión 
con la democracia local, sino con la eficiencia del sector público

3. El modelo neodesarrollista -o productivo con inclusión social- se inicia sobre fines del siglo XX, desplegado por gobiernos nacionales de izquierda. Su irrupción, es simultánea al desafío latinoamericano de avanzar en profundización democrática, y simultánea al reconocimiento y optimismo sobre la gobernanza como paradigma alternativo tanto para las ciencias sociales (académico) como para la gestión pública (político).

Por tanto, neo-desarrollismo, profundización democrática, y gobernanza, forman una triada que se interrelacionan y sirven de potencial marco analítico para comprender las pautas actuales de desarrollo, el tipo de Estado que se pretende instaurar, y la lógica de las políticas públicas.

La profundización democrática refiere a democratizar la democracia mediante políticas y procesos, para que ciudadanos participen informadamente en elecciones transparentes, influyan en decisiones políticas, y exijan responsabilidad a los gobernantes, y estos decidan respondiendo a la voluntad popular.

El interés está en cómo los ciudadanos adquieren capacidades para acceder a mecanismos de poder y elevar la calidad de la representación. Se trata de ir de una democracia electoral a una democracia de ciudadanía, no solo de libertades y derechos a elegir gobernantes, sino de derechos civiles y sociales universales y efectivos.

Es pasar a concebir al ciudadano como agente capaz de tomar decisiones relevantes para el bien público, como proponer, exigir y controlar. Por tanto, profundizar la democracia es hacerla real, es redistribuir recursos políticos y socioeconómicos hacia las mayorías, para que co-gobernara orientando recursos y políticas en función de sus intereses y necesidades.

\subsection{Conceptos claves para el paradigma de la gobernanza}

\subsubsection{Coordinación intersectorial e intergubernamental.}

En este contexto, hay un número creciente de problemas complejos, y una ciudadanía exigente que demanda más y mejores soluciones y servicios públicos. Pero esto no se soluciona única y simplemente aumentando el gasto público, requiere la articulación de diferentes conocimientos y diferentes actores (Zurbriggen y Travieso, 2016).

Así, la coordinación intersectorial y entre niveles de gobierno, se justifican no 
sólo en relación a la racionalidad fiscal, sino por la convicción de que los problemas sociales son multicausales y multidimensionales, y su superación amerita abordajes intersectoriales y multinivel que garanticen la integralidad en las políticas públicas.

Implica que los sectores se pongan de acuerdo para actuar conjuntamente a fin de lograr un cambio respecto de la situación inicial. La idea central remite a la integración de sectores y niveles gubernamentales como promesa de integralidad en la intervención sobre problemas sociales (Cunill Grau, 2014).

Esto implica un cambio en el plano epistemológico, apoyado en el pensamiento complejo (de autores como Morin, 2007) dado que se sostiene en la creciente conciencia de que la mayoría de los problemas que deben enfrentar los gobiernos son problemas con muchos factores causales, que conducen a objetivos de políticas en conflicto y desacuerdos acerca de causas y soluciones apropiadas, lo que requiere abordajes más interactivos entre disciplinas o especializaciones (Cunill Grau, 2014; Guy Peters, 2007).

\subsubsection{Participación ciudadana}

En este contexto, la concepción sobre la participación ciudadana está vinculada a lo que se espera del Estado, de la sociedad, a la relación entre ambos, y a la nueva concepción de ciudadanía. En esta etapa, el Estado asume responsabilidad central pero no monopólica, sino que también es necesaria una sociedad organizada y movilizada, capas de proponer, controlar, y reclamar, así como aportar recursos para darle mayor amplitud a la producción de bienes colectivos.

La ciudadanía no se concibe reducidamente relacionada al derecho al voto, sino también a otras dimensiones que conciban a cada persona y grupo como agente y sujeto de derecho y transformación. Una sociedad con mayor organización, movilización, y participación como forma de incrementar la probabilidad de que las decisiones de gobierno representen la voluntad de los ciudadanos.

El compromiso ciudadano favorece a que las personas dejen de ser dependientes de líderes y partidos, para ser ciudadanos conscientes de sus derechos y demandantes de políticas, lo que implica concurrir a ámbitos participativos y afiliarse a colectivos de la sociedad civil (Goldfrank, 2011). Así la participación ciudadana, no es un medio para asignar eficientemente los recursos y privatizar servicios, sino una forma de empoderar a la ciudadana en un rol de propuesta y control (Licha, 2002).

Así concebida, se espera que la participación ciudadana: a) fortalezca las 
organizaciones de la sociedad civil (mayores vínculos entre sí, más democráticas y eficaces; b) garantice y estimule el pluralismo (libertad de organización y expresión, y respeto a la autonomía de organizaciones; c) garantice y estimule la emergencia de nuevos actores (equidad de acceso a recursos económicos e institucionales;); apoyo público a lo que realizan d) desarrollo de mecanismos de responsabilidad pública (accountability), y control social sobre poderes existentes (gobierno, empresas y medios de comunicación (Delamaza, 2005).

\subsubsection{Descentralización}

Los procesos de descentralización suponen acercar la toma de decisiones entre niveles centrales y periféricos de gobiernos, y entre Estado y sociedad (Andreoli, 2012); y es clave para el neodesarrollismo, la democratización y la gobernanza. El nuevo modelo significa protagonismo del Estado en un contexto postfordista que exige flexibilidad para responder a diversas demandas por bienes y servicios públicos, lo que se traduce en la necesidad confluencia entre el nivel central y los subnacionales, que inevitablemente implica grados de descentralización funcional y territorial (Narbondo, 2013).

La descentralización adquiere relevancia vinculada a la democracia, como alternativa al centralismo autoritario. La hipótesis es que las autonomías territoriales permitirían el acercamiento del ciudadano y la sociedad civil a participar en asuntos públicos tanto desde la propuesta como desde el contralor (Mascareño, 2008); aumenta las posibilidades de representación y participación social dentro del esquema democrático (Gallichio y Camejo, 2005).

Desde la gobernanza, la descentralización introduce la perspectiva territorial y las relaciones entre niveles de gobierno. Ante la crisis del centralismo, la descentralización presenta la posibilidad de pensar desde una lógica horizontalterritorial, y abordar las problemáticas colectivamente en clave de una localización geográficamente delimitada (Arocena, 2006).

El territorio permitiría la construcción de objetivos coherentes y compartidos, y políticas resultantes del esfuerzo conjunto de organismos públicos y comunidades (Zubriggen, 2016). La descentralización implica una mayor densidad de transacciones y demandas sociales debido a la proximidad, que chocan con esquemas centralistas y homogeneizadores, dando lugar a nuevas incidencias decisorias de los actores 
territoriales (Nogueira, 2012).

\section{La descentralización territorial y la gobernanza en Uruguay}

Desde el 2005, Uruguay avanza hacia un nuevo Estado, un Estado presente en distintas áreas y con múltiples ámbitos descentralizados, de coordinación intersectorial y territorial, y participativos. Esto se ha denominado Transformación Democrática del Estado (TDE).

Esta TDE no es neutra, sino que fue iniciada desde un gobierno progresista perteneciente al Frente Amplio, que se ubica en lo que señalamos "marea rosa" en América Latina, o sea la emergencia del ascenso de las izquierdas como un fenómeno regional inédito (Pererira Da Silva, 2011), que están implementando un neodesarrollismo, llegan con la promesa de democratizar la sociedad y los Estados de los respectivos países (Goldfrank, 2007).

Textualmente ha señalado la Oficina de Planeamiento y Presupuesto (OPPPresidencia de la República) del periodo 2005-2010: "Se trata de una transformación democrática e integral del Estado para convertirlo en una estructura participativa, al servicio del ciudadano, en palanca del desarrollo productivo y en escudo de los débiles, y nada tiene que ver con las reformas del pasado. En efecto, esta transformación procura un Estado conductor, regulador, social y emprendedor; funcional a una agenda de desarrollo del país o, en otros términos, a un programa de crecimiento, distribución de la riqueza, modernización, democratización e inclusión social" (OPP, 2008: 5).

En esta TDE, es que han nacido muchas reformas: creación de los Municipios como consolidación del tercer nivel (local) de gobierno en el país; la creación de los Centros de Atención Ciudadana (CAC) con el objetivo de acercar la gestión de trámites principalmente a la población rural, las Mesas Interinstitucionales de Políticas Sociales con el objetivo de introducir la dimensión territorial a las políticas sociales, las Mesas de Desarrollo Rural para pensar el desarrollo de dicho medio con pequeños productores, productores familiares, y asalariados rurales, las Mesas Locales de Convivencia y Seguridad Ciudadana con el objetivo de ajustar las políticas de seguridad ciudadana a las necesidades y conocimiento de la población y acercar la rígida y jerárquica institución policial con la sociedad, entre otros.

En este artículo analizamos el funcionamiento de los tres últimos ámbitos 
señalados, correspondientes a los departamentos del noroeste del país (Paysandú, Salto y Artigas).

\section{Las Mesas Interinstitucionales de Políticas Sociales (MIPS)}

Las Mesas Interinstitucionales de Políticas Sociales (MIPS) tienen como finalidad articular el nivel central con el territorial en el desarrollo de las políticas públicas. Son la expresión del Gabinete Social y del Consejo Nacional de Políticas Sociales (CNPS) ${ }^{4}$ a nivel departamental (MIDES, 2014). Son presididas por Directores Departamentales del Ministerio de Desarrollo Social (MIDES) y formalmente deberían estar integradas por los representantes de mayor nivel jerárquico de los ministerios y organismos públicos que integran el CNPS. De manera que las MIPS son redes articuladas de organismos públicos que buscan sumar esfuerzos entre las instituciones para el abordaje de los principales problemas presentes en el territorio.

En relación a la dinámica de funcionamiento, puede verse que todas trabajan a partir de reuniones mensuales, en ellas se busca trabajar en torno a una agenda en cuya definición se advierte un protagonismo del Ministerio de Desarrollo Social (MIDES), quien convoca y realiza una agenda de puntos a tratar, sin perjuicio de que en los tres departamentos se manifiesta por parte de los representantes institucionales la posibilidad de agregar en el orden del día algunos temas de su interés.

En el caso de Artigas el protagonismo del MIDES es menos marcado que en los otros casos ya que en este departamento, la agenda está muy determinada por el Plan de Desarrollo Regional o Plan Artigas, que pre-existe a las mesas y que fue creado con la participación de las instituciones.

Se advierten valoraciones diferentes en relación a la contribución de este Plan de Desarrollo Regional en el departamento, por un lado, se valora como positivo el hecho de ya tener una agenda estratégica porque les permite concentrarse en los aspectos de la ejecución, por otro lado, hay discursos que marcan el hecho de que la instancia de las MIPS queda, en este contexto, reducida más bien a una rendición de cuentas, no generando un ámbito de intercambio fermental.

\footnotetext{
${ }^{4}$ Órganos creados en el 2005 por Presidencia de la República (gobierno central) con el objetivo de planificar e instrumentar las políticas sociales en el territorio nacional promoviendo la integralidad y la participación social.
} 
Por otra parte, algunas mesas en el país han optado por agregar a la instancia de trabajo general, realizada una vez al mes, una instancia de trabajo específico en mesas temáticas, tal es el caso de Salto en donde se definieron las siguientes mesas temáticas: de ruralidad, de educación, vivienda y hábitat, y Paysandú que ha avanzado en un Plan de Desarrollo Social con tres ejes y sus respectivo grupos de trabajo: educación, desarrollo productivo y hábitat.

Un aspecto que queda muy claro es que no existen problemas de autoridad en la coordinación o interinstitucionalidad, dado que en las tres mesas, tal vez Artigas en menor medida, muestran una alta legitimidad del MIDES en su rol de liderazgo de estos ámbitos, más allá de lo legal.

En los tres departamentos la toma de decisiones se realiza a través de acuerdos, en ningún caso se implementan otros sistemas laudatorios como la votación de los miembros, y en general se percibe que no hay mayores desacuerdos en relación a las problemáticas y las formas de abordarlas. Por lo general, las instituciones que tienen alguna injerencia en el tema generan acuerdos sobre la forma como se abordará la problemática.

Esta modalidad de trabajo que implica la deliberación y la búsqueda de acuerdos, en un contexto que además no genera demasiada conflictividad, es valorada positivamente por los representantes en los diferentes departamentos con algunas excepciones puntuales en donde se percibe al mecanismo como algo laxo y poco efectivo o vinculante.

Se observa un alto grado de consenso en la identificación de problemas y de acciones, sin embargo, eso puede estar atado a que el alto nivel de centralización no implica desenvolvimiento de recursos propios y la tarea es más sugestiva que resolutiva. Se podría decir que la no existencia de recursos propios para la coordinación y el hecho de que las bases territoriales de los ministerios, de los entes autónomos y de los servicios descentralizados (que integran las MIPS), más que expresiones de descentralización conforman espacios de desconcentración administrativa, contribuye a la existencia de acuerdos fáciles y poco conflictos, pero al mismo tiempo, como se verá más adelante, tiende a generar un espacio con poca ejecutividad, que tiene más buenas intenciones de lo que logra poner en marcha.

En el caso de Artigas, se menciona como una ausencia importante a las 
instituciones vinculadas a la Educación. En Salto, la ausencia cualitativamente más importante que aparece en los discursos es la de la Intendencia Departamental, la cual es visualizada como una institución con una presencia muy intermitente y en general con miembros técnicos y no dirigentes políticos.

En Paysandú, sin embargo, la Intendencia Departamental está presente pero la idea que sobrevuela con fuerza es que "está pero no está”, no tiene el protagonismo que tenía en el periodo anterior y participa con un miembro que no tiene poder de resolución, que básicamente tiene el rol de informar a la Intendencia de lo que sucede en las MIPS.

La idea que se esgrime como justificación de este rol secundario por parte del representante de la Intendencia en las MIPS, pasa por el lado de que en realidad la Intendencia no es un miembro pleno sino un invitado a participar. Debe tenerse en cuenta, sin embargo, que los miembros invitados tienen los mismos derechos que los otros, la diferencia estaría en que para ellos es opcional participar o no, mientras que para los miembros plenos es una obligación inherente a la función.

El peso del argumento de no sentirse un integrante pleno no parece demasiado contundente para justificar el escaso protagonismo de la Intendencia de Paysandú, sobre todo teniendo en cuenta que venían de un periodo anterior, bajo la conducción del Frente Amplio, donde habían tenido un protagonismo mucho mayor. Sí parece ser un argumento de peso la diferencia de color político con el gobierno nacional, aspecto que también es relevante en el caso de Salto. Este punto se retomará más adelante.

Extendido a los tres casos analizados, se observa que el predominio de la participación es de tipo técnico, esto es, participantes con un saber especializado pero sin facultad para tomar las decisiones en nombre de su institución.

Los hallazgos analizados permiten evidenciar con claridad la posición diferencial de los actores ante la lógica de la inter-sectorialidad y la coordinación. Para unos la coordinación es clave para su trabajo mientras para otros no, lo que genera un fenómeno de profesionalización de la gestión coordinada y cierta elitización en el dominio de los ámbitos.

En relación a la evaluación del funcionamiento de la MIPS, en Artigas existen visiones opuestas respecto a la ejecutividad-efectividad del ámbito, sin embargo, predomina una visión negativa de la MIPS en este punto, se la visualiza como un 
ámbito no ejecutivo, no resolutivo, muy poco operativo.

En el mismo sentido, en Salto se evidencia que la MIPS no es efectiva, no trasciende algunas coordinaciones puntuales, y es más bien informativa, con excepción de algunas comisiones de trabajo que son resaltadas muy positivamente como forma de trabajo de la MIPS en el departamento.

En Paysandú, se valora la capacidad de planificación a mediano plazo y la rápida construcción de una lógica o cultura de la interinstitucionaldad, sin embargo, también se observa en general la poca ejecutividad, operatividad y efectividad. Esa falta de ejecutividad junto a la concentración de temáticas que no representa el interés de la totalidad de las instituciones motiva algunas de las ausencias.

La percepción de la MIPS como un espacio no ejecutivo, que aparece con fuerza en los tres departamentos, debe analizársela teniendo en cuenta lo que se ha dicho anteriormente, que quienes participan de este espacio son mayormente jerarcas departamentales (o eventualmente regionales) de organizaciones nacionales que mantienen una fuerte centralidad en la toma de decisiones, por lo cual, no todos los problemas se pueden resolver con acuerdos de las mesas, sino que requieren negociaciones a otro nivel.

En este escenario, se hace aún más relevante la presencia activa de la Intendencia Departamental, disponiendo de recursos y con capacidad decisoria dentro de sus áreas de competencia.

En lo que respecta al aspecto motivacional, se puede decir que en Artigas pese a la ya dicha percepción negativa, las motivaciones a participar se explican por la persistencia de una credibilidad sobre la interinstitucionaldad como forma de resolver problemas comunes, más allá de los resultados.

También se observa cierta "elitización" o control del espacio por parte del MIDES y la Intendencia Departamental. En Salto, las motivaciones son muy específicas y compartidas, y tienen que ver con: entender que los problemas sociales son multicausales, multidimensionales y su naturaleza exige intervenciones multisectoriales y multidisciplinares.

Por su parte, en Paysandú, en la motivación a participar se advierte un híbrido entre la obligatoriedad legal o por designación, que son quienes no se involucran activamente, y por la eficiencia que implica la interinstitucionaldad en relación a no 
superponer esfuerzos y recursos, ampliar la mirada sobre la realidad social del territorio, y abordar problemas multicausales desde soluciones multisectoriales, que son quienes se involucran activamente. Un aspecto observado es que la sectorialidad aún pesa sobre la intersectorialidad.

Por otro lado, las MIPS actúan más como ámbitos de territorialización de políticas nacionales que como ámbitos de políticas públicas territoriales, es decir más como ámbitos para la mejor implementación departamental de lo nacional que como ámbitos de iniciativa y ejecuciones propias de lo departamental.

Si a esto le sumamos que los titulares de las Intendencias Departamentales son electos por la ciudadanía y que éstas son jurídica, técnica y presupuestalmente autónomas, estamos ante un escenario donde existe un alto nivel de descentralización en la implementación más que en el diseño (agendas o planes departamentales) de políticas.

Se puede ver que en Artigas, la Intendencia aparece como un miembro que junto al MIDES co-lidera el ámbito, lo que se explica por su sintonía política-partidaria con el gobierno nacional. Se debe tener en cuenta que la mayor parte de los integrantes de la MIPS son representantes locales de los Ministerios, por lo cual, el color político del gobierno central es un aspecto clave para entender la vinculación de la Intendencia.

Este escenario de colaboración entre estos dos niveles de gobierno no estará presente en los otros dos casos. Aquí pudo observarse una Intendencia activa que asignó recursos e implementó políticas sociales en el marco del espacio. Respecto a la sociedad civil, se advierte que ésta tiene un involucramiento muy débil, se remitió a co-elaborar el Plan Artigas $^{5}$ (utilizado por la MIPS), pero no se le convoca ni se procura su participación en la implementación y evaluación de dicho plan. Se advierte un alto desconocimiento de la sociedad respecto a la MIPS.

En Salto, la Intendencia aparece sistemáticamente mencionada en los discursos como la gran ausencia de la MIPS, una ausencia que además se denuncia como un debe, un déficit que facilitaría sobremanera el trabajo en cuanto a su rol de logística, y de

\footnotetext{
${ }^{5}$ Consiste en un documento estratégico básico pensado para ejecutarse en el periodo comprendido entre 2012 y 2015. Este plan fue desarrollado a iniciativa de la Intendenta Patricia Ayala y contó con la participación de los principales actores sociales y políticos departamentales.
} 
gobierno de cercanía con la ciudadanía.

Aparece fuerte la idea de que antes (en el periodo anterior) participaba más, por lo que se puede deducir que la ausencia refiere a diferencias político-partidarias entre Gobierno Departamental y Gobierno Nacional. Cuando se analiza la coordinación entre niveles esta dimensión política, vinculada a la coincidencia (o no) de colores de partidos, suele impregnar todo el esquema de coordinación (Repetto, 2007) y en los casos estudiados se ve con claridad.

En relación a la vinculación de la MIPS de Salto con la sociedad civil, se puede decir que la participación se visualiza como muy pobre, puntual y esporádica.

En Paysandú, la Intendencia participa pero de forma testimonial, sin capacidad de asumir compromisos en tiempos reales. Se identifican relatos del tipo de "ellos" y "nosotros", la Intendencia de Paysandú aún no se siente parte de la MIPS y el resto de la Mesa la mira con desconfianza. En los discursos surge de forma exclusiva que el hecho de predominar partidos distintos puede explicar el fenómeno.

\section{Las Mesas de desarrollo rural (MDR)}

Las Mesas de Desarrollo Rural (MDR) fueron creadas en el año 2007, con el cometido de promover la participación de los actores que viven y trabajan en el medio rural, en la identificación de sus necesidades y la elaboración de la agenda de desarrollo para su zona de influencia. Estos espacios de gestión participativa, tienen entre sus propósitos la democratización de las relaciones entre el Estado y la sociedad civil organizada, por lo que su instrumentación requiere la puesta en marcha de instrumentos que impulsen la planificación y el control de la ciudadanía en el diseño de las estrategias de desarrollo así como en el manejo de los recursos para llevarlas a cabo.

Las Mesas de Desarrollo Rural aunque comparten problemáticas y dificultades comunes, adquieren distintas modalidades de funcionamiento según departamento. Las organizaciones de productores de Artigas y Salto se agrupan en tres Mesas de Desarrollo que funcionan en las localidades o centros poblados de referencia, mientras que en Paysandú es un único espacio (es decir una única Mesa) a nivel departamental en el que participan todas las organizaciones del departamento, rotando sistemáticamente el lugar de encuentro. 
La asistencia en el caso de Salto y Paysandú, es evaluada como satisfactoria, mientras que en las Mesas de Artigas se señalan ausencias significativas como el Movimiento de Erradicación de la Vivienda Rural Insalubre (MEVIR), el MIDES y Alcoholes del Uruguay (ALUR) que son instituciones claves en el departamento.

Un aspecto que resulta interesante de los discursos que reflejan un gran vacío en términos de participación ciudadana, es la contradicción entre una aparentemente "buena asistencia" y el rol pasivo y meramente receptivo de las organizaciones, mediado a su vez por la irregularidad del funcionamiento de espacio.

Es decir, los discursos revelan diferentes niveles de involucramiento y apropiación del espacio en el interior de cada MDR, destacándose en todos ellos, sin embargo, una valoración altamente positiva de la propuesta en términos de mejoramiento de las posibilidades de crecimiento y desarrollo de cada organización y de atención a las demandas de los pobladores rurales en general.

A diferencia de lo que sucede en Artigas, la MDR de Paysandú tiene un funcionamiento regular, con una asistencia de 35 personas aproximadamente, las que si bien representan a gran parte de las organizaciones, en algunos casos se da una sobrerrepresentación de alguna de ellas.

Por su parte en el caso de Salto ${ }^{6}$, en las tres Mesas se destaca un funcionamiento regular con una asistencia promedio de 20 personas, dependiendo la zona de influencia. No obstante en una de ellas (Basalto Profundo), se señala una disminución en la asistencia ante logros de planteos individuales.

Las manifestaciones relevadas dan cuenta de que las reuniones son mensuales en los tres casos, con un nivel de asistencia de regular a bueno; en general el orden del día se elabora desde el Ministerio de Ganadería Agricultura y Pesca (MGAP) en función de la planificación anual elaborada en cada Mesa y de las demandas que éstas van planteando; en el caso de Artigas, el funcionamiento es sobre las demandas, sin planificación previa.

6 En el caso de Salto, funcionan desde el año 2009, tres MDR: 1. Producción Intensiva - Cordón hortofrutícola, producción avícola, producción caprina; 2. Basalto Profundo; 3. Basalto Superficial. En los tres casos la frecuencia de reuniones es mensual, con día fijo de encuentros pero con una rotación de los lugares donde se realizan entre las sedes y/o localidades de las organizaciones anfitrionas. 
Asimismo se pueden incorporar otras temáticas tanto por parte de las organizaciones como las instituciones -que asisten con distinto nivel de asiduidad a los espacios-, dependiendo de las temáticas a trabajar. Las resoluciones son discutidas en el ámbito de la Mesa y articuladas con las instituciones involucradas en cada problemática o planteo.

Ante demandas puntuales para cuya solución están estipulados programas específicos, existe una respuesta relativamente rápida; no obstante cuando se requieren acciones interinstitucionales que exceden a las organizaciones, la respuesta lenta y se diluye en el tiempo (dependiendo de la "capacidad de presión" de las organizaciones y de cada Mesa).

En este sentido y haciendo referencia a los efectos que la respuesta y la norepuesta generan en términos de participación, se visualizan, por un lado, un alejamiento de aquellas organizaciones que una vez satisfecha su demanda, se desvinculan del espacio, y por otro, más allá de las valoraciones con respecto a la forma y tiempo en que desde los organismos competentes se da (o no se da) seguimiento a los planteos realizados, una permanencia de las organizaciones que destacan los logros colectivos que desde las Mesas han alcanzado, trascendiendo sus demandas particulares.

Es decir, se podría argumentar que la obtención de respuestas favorables en términos individuales, no genera procesos de apropiación del espacio como sí lo hacen los logros colectivos, más allá de la lentitud de la respuesta y/o del beneficio no individualizado que generan algunas de ellas.

El proceso de toma de decisiones en las MDR se realiza generalmente por consenso y eventualmente por votación de mayorías. Los asuntos o temáticas sobre los que se procesan tales decisiones refieren fundamentalmente a la emisión de avales para la presentación de proyectos productivos y/o de fortalecimiento institucional, la priorización de las necesidades en el territorio que forman parte de la agenda de cada Mesa, y la ejecución de la política en su etapa final con una autonomía muy relativa que se disipa en aquellos espacios cuya dinámica de funcionamiento es irregular e inestable (como lo es el caso de las Mesas de Artigas).

Por otra parte, en relación a la vinculación de las MDR con el gobierno departamental, de las entrevistas realizadas se desprende que en general la Intendencia es vista como un actor con presencia activa en los espacios, más allá de los resultados 
de su gestión en el territorio. Caso contrario sucede con los Municipios cuya participación e involucramiento con la propuesta, es considerada como muy reducida o casi nula.

No obstante ello, en las localidades más alejadas de los centros urbanos y por ende, con un acceso muy restringido a los servicios sociales, se visualiza una fuerte vinculación con la comunidad local; tal es el caso de una de las Mesas de Artigas (Pueblo Sequeira), donde los actores perciben el espacio conformado en torno a la Mesa, como el ámbito desde el cual canalizar sus demandas e inquietudes.

\section{La Seguridad Ciudadana y la Convivencia}

Robert Castel esboza algunas funciones que debe cumplir el Estado como asegurador y protector tanto de los derechos civiles como de los derechos sociales con el propósito de no derivar en la inseguridad y la disociación social, haciendo foco principalmente en la cohesión social como eje central de su función (Gómez, 2013).

En este sentido, se configura en primer lugar el papel que debe desempeñar el Estado de Derecho, esto es, acabar con la inseguridad civil, es decir, con aquellas amenazas a la integridad de las personas y de los bienes, tales como: la delincuencia, el robo, la violencia, la criminalidad, etc. (Gómez, 2013).

Por otra parte para el PNUD la seguridad humana se vincula a la noción capital social:

"La seguridad humana se asienta en el valor que alcanza el capital social logrado en una sociedad, definido como una acumulación de experiencias compartidas que han ido estableciendo redes sociales, instituciones informales, lazos sociales, valoraciones comunes de lo que es positivo o negativo para el desarrollo, convivencia e identidad nacional” (PNUD apud Gómez, 2013).

De acuerdo a lo estipulado por el Ministerio del Interior, las Mesas locales para la convivencia y la seguridad ciudadana son un espacio de participación e intercambio entre diferentes actores sociales para trabajar en los temas relacionados con la seguridad y la convivencia desde una mirada local (Ministerio del Interior, 2015).

En términos de diseño estos espacios estarían integrados por la Seccional del barrio con la Policía Comunitaria, representantes de los Consejos Vecinales, representantes de institucionales a nivel local, vecinas/os, que deseen participar 
activamente en el compromiso de mejorar la seguridad y convivencia.

Los propósitos de la mesa de acuerdo a lo dispuesto por el Ministerio del Interior (2015) consisten en identificar problemas de convivencia y seguridad, para establecer prioridades y acuerdos y programar acciones en conjunto, logrando compromisos entre las instituciones y los vecinos buscando las posibles soluciones a los problemas.

Respecto a quienes participaban, los entrevistados decían lo siguiente:

"Referentes de Comisiones vecinales, de instituciones educativas, de instituciones deportivas, en el caso nuestro referentes del Ministerio de Desarrollo Social, todos los que tengan alguna incidencia o alguna actividad en el área de influencia son invitados, después participa quien lo desea” (Sociedad Civil, Salto).

La pluralidad de actores que se involucran en estos espacios parece tener una doble explicación: lo primero es la flexibilidad de integración. No existe en ningún caso una mención reglamentaria a quienes deben integrarse por tanto todo aquel que se sienta tocado por el tema, más aún si tiene algún tipo de gimnasia de participación se siente con la potestad de integrarla.

Lo segundo es que el voluntarismo con que se movió el Estado en este caso genera un cierto stress para llamar a todo aquel que esté disponible, aunque no se tuviera claro para qué.

Otra dimensión interesante y complementaria con lo anterior es la percepción del Área de influencia de la Mesa. Muchas veces el exceso de reglamentación termina por burocratizar el mecanismo de participación ahogando la iniciativa de la población pero cuando la falta de una estructuración y de liderazgo es tan grande esto se vuelve un boomerang para la instrumentación de la política. O se aclara hacia dónde vamos, quiénes somos y para qué estamos o lo más lógico es que no funcione o se torne inútil el instrumento, ese parece ser la consecuencia en este caso.

En este sentido es razonable pensar que la gente optimice el uso de su propio tiempo, salvo quienes de alguna manera están comprometidos institucionalmente con el proceso, el ciudadano de a pie, evaluará, y con razón, la eficacia de su actuación respecto a los propósitos declarados o percibidos del instrumento.

Como se vio anteriormente, son mesas de seguridad ciudadana pero las razones de la intervención de los vecinos son múltiples, se trata de hacer oír su voz en función de los problemas cotidianos no siempre restringidos a la convocatoria. Tener un 
espacio, un lugar y alguien que oiga, esa es su primera motivación.

El funcionamiento del mecanismo generó un tipo de participante rentado y en otros casos vecinos comprometidos, pero fundamentalmente reducido. En otras palabras con un funcionamiento irregular y de escasa entidad sólo asociado a quienes obtienen un beneficio pecuniario con el funcionamiento.

Es necesario comentar que la percepción sobre la construcción de la agenda de las reuniones, de acuerdo a los entrevistados, la conformaban los referentes institucionales, o sea, nuevamente se observa un liderazgo débil del Ministerio y las fuerzas de seguridad y si este mecanismo es cooptado por las demás instituciones con mayor práctica en el terreno: MIDES, Comisiones de vecinos, etc...

Esto tiene una doble interpretación, por un lado es una ventaja, ya que los temas resultan de las necesidades de los vecinos manifestadas no solamente en ese espacio que probablemente no usaban o que las necesidades expresadas fueran las estrategias políticas de otras instituciones incluso ministeriales, lo que hace absolutamente inocuo el uso del recurso, ya que si funciona otro mecanismo, no es necesaria la creación de uno nuevo con el consiguiente derroche de energía social que ello significa.

\section{Conclusiones}

Para concluir en cuanto al funcionamiento de las entidades, se visualizan algunas similitudes y algunas diferencias entre los tres dispositivos estudiados en los tres departamentos.

Las que aparecen con más personalidad son las MIPS que marcan una forma de trabajo a nivel territorial con una amplia participación de diferentes instituciones del Estado (con un vínculo muy débil con la sociedad civil) y solamente en Artigas es que ceden parte de su impronta a la existencia de un Plan Departamental de Desarrollo.

Las Mesas que sostienen una imagen y funcionamiento más sectorializadas y por ende más centralizadas son la MDR, donde el papel del Ministerio de Ganadería Agricultura y Pesca es protagónico y con una impronta específica, incluso asignando recursos técnicos al funcionamiento del mecanismo.

Las mesas con una trayectoria voluntarista y con alto nivel de improvisación son las de Seguridad Ciudadana, es donde la acción gubernamental está menos definida y que se traduce en el funcionamiento y sostenibilidad del mecanismo haciéndolo frágil y 
con pocas posibilidades de incidencia. Incluso en Artigas no se conformaron nunca.

Como sugerencia inicial y a la luz de este estudio, es posible afirmar la necesidad de reformulación de la política de descentralización, participación y de horizontalización a nivel territorial.

Esta debe implicar la fractura de la lógica histórica de sectorialización vertical y apostar fuertemente al fortalecimiento de los actores territoriales con posibilidades de accionar. Esta transformación no debe olvidar que la política de participación voluntaria se sostiene en función de los éxitos y logros de la misma siendo vital la constatación a nivel de los implicados de ellos.

Por otro lado, se debe abordar la simplificación de los mecanismos de participación en virtud de la reiteración en diferentes ámbitos de las mismas reivindicaciones. Los actores son muchas veces los mismos y las reivindicaciones igual, por tanto, simplificar el funcionamiento e incluso combinarlo con otras modalidades menos estables y más flexibles parece ser un buen camino a seguir.

Sin duda que el trabajo que se presenta aquí es inicial y ameritará mayor desarrollo en los próximos meses con vistas a enriquecer la reforma planteada, pero como un esbozo introductorio resulta por ahora satisfactorio. 


\section{Referencias}

Abal, J. M. (2011). "La reforma del Estado y el fortalecimiento de las instituciones públicas", en: Amaya Paula y otros (Coord.) El Estado y las políticas públicas en América Latina. COPPPAL, AECID, Universitaria la Plata. (Págs. 17 a 36). La Plata - Argentina

Adelantado, J. (2009). "Por una gestión "inclusiva" de la política social". En Chiara, M.; Di Virgilio, M. (org.) Gestión de la política social. Prometeo Libros: Buenos Aires.

Aguilar, L. F. (2000). La hechura de las Políticas. Ed. Miguel Ángel Porrúa: México.

Barrera, A. (2009). "Innovación política y participación ciudadana. Tendencias democráticas en los gobiernos locales". En H. Poggiese y T. Cohen Egler (comp.) Otro desarrollo urbano: ciudad incluyente, justicia social y gestión democrática. 1a ed. Consejo Latinoamericano de Ciencias Sociales: Buenos Aires.

Cabrero, E. (2006). Acción pública y desarrollo local. En Revista de Gestión y Política Pública, vol. XV, núm. 2.

Cunill, N. (2005). La Intersectorialidad en el Gobierno y Gestión de la Política Social. Documento preparado para el Diálogo Regional de Políticas, Red para la Reducción de la Pobreza y la Protección Social. Washington D.C.: BancoInteramericano de Desarrollo.

De Mattos, C. (1990). La descentralización, ¿Una nueva panacea para impulsar el desarrollo local? En Estudios Regionales, N²6. 49-79.

Delamaza, G. (2005) Tan lejos, tan cerca: Políticas públicas y sociedad civil en Chile. LOM: Santiago de Chile . Disponible en:

Evans, P. (1996). "El Estado como problema o solución”. En Revista Lua Nova No 28/29. San Pablo - Brasil.

Franco, R. (1996). Los paradigmas de la política social en América Latina”. Santiago de Chile: CEPAL.

García, D y Peirano, M. (2011).El modelo de desarrollo con inclusión social. La estrategia a mediano plazo. Buenos Aires: CICCUS-IDISA-FLACSO.

Garretón, M. (2004). América Latina en el siglo XXI. Hacia una nueva matriz sociopolítica. Santiago de Chile: LOM Ediciones.

Gómez, L. (2013). Seguridad ciudadana: Enfoque desde la seguridad comunitaria. Tesis de grado, Licenciatura en Ciencias Sociales- Regional Norte, Udelar.

http://pdba.georgetown.edu/Security/citizensecurity/uruguay/documentos/institucionalid ad.pdf

http://www.republica.com.uy/seguridad-ciudadana/

Kliksberg, B. (1999). "Seis Tesis no convencionales sobre participación". En Revista de Estudios Sociales. № 4. Universidad de Los Andes: Bogotá.

Licha, I. (2002). "El debate actual sobre desarrollo participativo". En Licha, Isabel (editora). Gerencia Social en América Latina. Banco Interamericano de Desarrollo (BID). Washington D.C- Estados Unidos.

Licha, I., Molina, G. y Repetto, F. (2007). La coordinación de las intervenciones públicas en los problemas sociales: desafíos y estrategias. Caracas: CLAD.

Lucero, M. (2009). "Participación ciudadana". En N. Aquín (comp.) Políticas Públicas, Derechos y TS en el Mercosur". Buenos Aires.

Marsiglia, J., Pinto, G. y Galicchio, E. (2012). "Investigación sobre Descentralización y 
autonomía local en los Estados miembros del MERCOSUR" Informe de Sistematización de los cuatro estudios nacionales (Paraguay, Uruguay, Argentina y Brasil). Montevideo: CLAEH.

Martínez, R. (2012). La institucionalidad y gobernanza: ¿nuevas perspectivas para la gestión pública? En Aportes para el Estado y la Administración gubernamental. $N^{o} 30$, págs. 53-68.

Mayntz, R. (2001). "El Estado y la sociedad civil en la gobernancia moderna”. Revista del Centro Latinoamericano de Administración para el Desarrollo (CLAD).

Ministerio de Desarrollo Social (2014). Mesas Interinstitucionales de Políticas Sociales (MIPS) Reporte del Sistema de Información. Periodo 2011 - 2013. MIDES. Montevideo-Uruguay.

Ministerio del Interior (2015). Mesas Locales para la Convivencia y Seguridad Ciudadana Disponible en: https://www.minterior.gub.uy/index.phpoption=com_content\&view=article\&id= $\underline{102}$

Moreira, C. y Veneziano, A. (1995). Intergovernmental relations in Uruguay. En Nagel, Stuart (Comp.). Political Reform in Developing Nations. Illinois University Press.

Morin, E. (2007). Introducción al Pensamiento Complejo. Editorial Gedisa. España Oficina de Planeamiento y Presupuesto (2008) Transformación democrática del Estado y nueva relación con la ciudadanía. OPP - Presidencia de la República Oriental del Uruguay. Montevideo-Uruguay

Oficina de Planeamiento y Presupuesto (2008). Transformación democrática del Estado y nueva relación con la ciudadanía. Montevideo: OPP - Presidencia de la República Oriental del Uruguay.

Repetto, F. (2005). "La dimensión política de la coordinación de programas y políticas sociales: una aproximación teórica y algunas referencias prácticas en América Latina”, en Repetto, Fabián (ed.) La gerencia social ante los nuevos retos del desarrollo social en América Latina. INDES Ciudad de Guatemala - Guatemala.

Repetto, F. y Fernández, J. (2012). Coordinación de políticas, programas y proyectos sociales. Buenos Aires: CIPPEC.

Sanseviero, R. (2005). Institucionalidad Pública en el ámbito de la Seguridad Ciudadana; entre las intenciones y las tensiones en Uruguayo.

Serra, A. (2004). "La gestión transversal: expectativas y resultados". Ponencia presentada en: IX Congreso Internacional del CLAD, SOBRE LA Reforma del Estado y de la Administración Pública. Madrid - España (2-5 noviembre 2004)

Veneziano, A. (2005). Reflexiones sobre una reforma del estado orientada al ciudadano: la descentralización participativa de Montevideo. Ed. Inap: Madrid.

Veneziano, A. (2009). Descentralización, Desarrollo Local, Participación Ciudadana y Reforma del Estado: una vinculación pendiente. Montevideo: FCS- UdelaR CSIC. Ediciones ORBE.

Wright, D. (1988). Understanding intergovernmental relations. California: Brooks/Cole Publishing Company.

Zurbriggen, C. Morales, S. y Rak, A. (2010). "De lo sectorial a lo transversal: hacia una nueva forma de gestión pública". En Mancebo. M.E y Narbondo P (Coords.) Reforma del Estado y políticas públicas en la Administración Vázquez. Editorial Fin de Siglo. Montevideo - Uruguay. 